\title{
TESTING OF DIODE-CLAMPING IN AN INDUCTIVE PULSED PLASMA THRUSTER CIRCUIT
}

\author{
Alexandra Toftul ${ }^{1}$, Kurt A. Polzin ${ }^{2}$, and Adam K. Martin ${ }^{3}$ \\ NASA - George C. Marsahll Space Flight Center, Huntsville, AL 35812 \\ and \\ Jerry L. Hudgins ${ }^{4}$ \\ University of Nebraska-Lincoln, Lincoln, NE 68588
}

\begin{abstract}
Testing of a $5.5 \mathrm{kV}$ silicon (Si) diode and $5.8 \mathrm{kV}$ prototype silicon carbide ( $\mathrm{SiC}$ ) diode in an inductive pulsed plasma thruster (IPPT) circuit was performed to obtain a comparison of the resulting circuit recapture efficiency, $\eta_{r}$, defined as the percentage of the initial charge energy remaining on the capacitor bank after the diode interrupts the current. The diode was placed in a pulsed circuit in series with a silicon controlled rectifier (SCR) switch, and the voltages across different components and current waveforms were collected over a range of capacitor charge voltages. Reverse recovery parameters, including turn-off time and peak reverse recovery current, were measured and capacitor voltage waveforms were used to determine the recapture efficiency for each case. The $\mathrm{Si}$ fast recovery diode in the circuit was shown to yield a recapture efficiency of up to $20 \%$ for the conditions tested, while the SiC diode further increased recapture efficiency to nearly $30 \%$. The data presented show that fast recovery diodes operate on a timescale that permits them to clamp the discharge quickly after the first half cycle, supporting the idea that diode-clamping in IPPT circuit reduces energy dissipation that occurs after the first half cycle.
\end{abstract}

\section{Nomenclature}

$C=$ capacitance

$E_{C i} \quad=$ initial capacitor energy

$E_{C f} \quad=$ final capacitor energy

$i(t) \quad=$ loop current

$I_{d R M} \quad=$ peak reverse recovery current

$I_{F, A V} \quad=$ mean forward current

$Q_{r r} \quad=$ reverse recovery charge

$t_{o} \quad=\quad$ time of initial current zero crossing

$t_{r r} \quad=$ reverse recovery time

$V_{C}(t)=$ capacitor voltage

$V_{C i} \quad=$ initial capacitor voltage

$V_{C f} \quad=$ final capacitor voltage

$V_{R R M} \quad=$ maximum reverse blocking voltage

$z_{o} \quad=$ electromagnetic decoupling distance

$\eta_{r} \quad=$ recapture efficiency

\footnotetext{
${ }^{1}$ Flight Sy stems Engineer, Sy stems Integration Branch, Vehicle Integration Depatment, alexandra.toftul@ nasa.gov, Member AIAA.

${ }^{2}$ Propulsion Research Scientist, Propulsion Research and Technology Applications Branch, Propulsion Systems

Department, kurt.a.polzin@ @asa.gov, Associate Fellow AIAA

${ }^{3}$ Physicist, Propulsion Research and Technology Applications Branch, Propulsion Sys tems Department, adam.k.martin@nasa.gov

${ }^{4}$ Profes s or and Chair, Department of ElectricalEngineering, jhudgins2@unl.edu
} 


\section{Introduction}

TNDUCTIVE pulsed plasma thruster (IPPT) circuits, such as those needed to operate the Pulsed Inductive Thruster (PIT), are required to quickly s witch high-voltage capacitor banks through an inductive acceleration coil at current levels on the order of at least $10 \mathrm{kA}$ operating on a period of 1-10 $\mu \mathrm{s} .{ }^{1}$ In most IPPTs, the required voltage holdoff between pulses and the high current levels during pulses have led to the use of spark gaps in discharging the capacitor bank. Recentavailability of fast solid-state $s$ witching devices capable of both holding off high voltages and conducting increasingly high current has made it possible to consider the use of these s witches in modern IPPTs. In addition, the production of a pre-ionized gas prior to the discharge through the acceleration coil permit a reduction in the required discharge energy per pulse for electric thrusters of this type, relaxing the switching requirements for these thrusters. ${ }^{2}$ Solid state s witches offer the advantage of greater controllability, reliability, and longer lifetime, as well as decreased drive circuit dimensions and mass relative to spark gap s witches.

The capacitor bank, inductive acceleration coil, and external resistance and inductance of an IPPT pulse circuit can be modeled as a simple series resistive-inductive-capacitive $(R L C)$ circuit. Once the switch is closed, the natural energy exchange between the reactive components will cause the stored energy in the circuit to os cillate between the capacitive and inductive elements until it has been used to accelerate a plas ma or resistively dissipated in the circuit. It has been previously shown ${ }^{3,4,5}$ that themost efficient coupling of energy into the plas ma current sheet of a PIT occurs during the first current half-cycle of the capacitor discharge, when the coil current and current rise rate are maximum and the plasma is spatially closest to the coil. After this time, the current sheet has moved away and is largely electromagnetically decoupled from the inductive coil, leaving the remaining energy in the circuit to be resistively dissipated while performing no work. This energy could be recaptured in the capacitor bank and used in part for future pulses if the current flow in the circuit can be interrupted after the first half-cycle of the discharge.

Energy recapture can be achieved by placing a fast turn-off diode in series with the main switch. In this case, higher circuit recapture efficiency, $\eta_{r}$, defined as the percentage of the initial charge energy remaining in the capacitor bank aftercurrent stops flowing, may be achieved by using a diode that is able to transition, or 'switch,' from the forward conducting state ('on' state) to the reverse blocking state ('off' state) in the shortest amount of time, minimizing current ringing and s witching losses. This is known as the reverse recovery time and is dependent on the diode material and internal structure. Investigating the effect of diode reverse recovery on recapture efficiency could potentially lead to the development of novel, high-efficiency IPPT circuit topologies.

In Section II, we describe the motivation for more efficient utilization of electrical energy in IPPT pulse circuits and propose how this can be improved through the use of fast recovery diodes (FRDs). Described in Section III is the design and fabrication of the small-scale IPPT used as a tes tbed for the several types of diodes. In Section IV, we present the experimental setup used to test several different diode types and quantify their ability to interrupt the oscillating current in the pulse circuit. Comparative data demonstrating the capability to clamp the circuit with different types of diodes, recapturing otherwise dissipated energy in the capacitor bank, are presented in Section V.

\section{Inductive Pulsed Plasma Thruster Circuit Topology}

After an IPPT completes the first half-cycle of the discharge, during which inductive acceleration of a plasma occurs, any further oscillation of the dis charge current causes excess heat generation and power waste, both of which are highly undesirable in a space environment. In the absence of convective cooling, heat removal from circuit components becomes a major concern, particular for electrical components that may fail above a maximum rated operating temperature that is still quite low in terms of the ability to radiate that energy to space. Heating must be managed using heat sinks, which can add significant mass to a system. Similarly, inefficient use of electrical energy neces sitates a larger, more massive, and typically more costly electrical power system (EPS). Furthermore, voltage reversals in the circuit have been shown to adversely affect capacitor lifetime. ${ }^{6}$ In the absence of a snubber circuit, the high reverse voltage rise rate can stress the solid state s witch, reducing the long-term reliability of the device. As IPPTs are designed for long-duration, deep-space mis sions that may require the pulse circuit to survive for upto $10^{10}$ discharge $\mathrm{cycles}^{7}$, these reductions in component reliability and lifetime are problematic. While the use of one or more redundant circuits will reduce the probability of a system failure, the associated increase in thruster mass, volume, cost, and complexity are clearly undesirable. 
To address these is sues, a FRD can be placed in series with an IPPT's switch. The purpose of the diode is to clamp the current in the circuit following the first half-cycle of the discharge. Two PiN structure powerdiodes ${ }^{8}$ were tested in the circuit in Fig. 1 to determine the effect of the diode material on the IPPT circuit recapture efficiency, $\eta_{r}$. The two series diodes tested were a silicon $(\mathrm{Si}) \mathrm{ABB}$ 5SDF 02D6004 PiN power diode rated for $5.5 \mathrm{kV}$ and $175 \mathrm{~A}$ (Fig. 2a), and a prototype silicon carbide (SiC) PiN diode from Cree, Inc. rated at $5.8 \mathrm{kV}$ (Fig. 2b). While a current rating for the prototype device is not available, thermal analysis

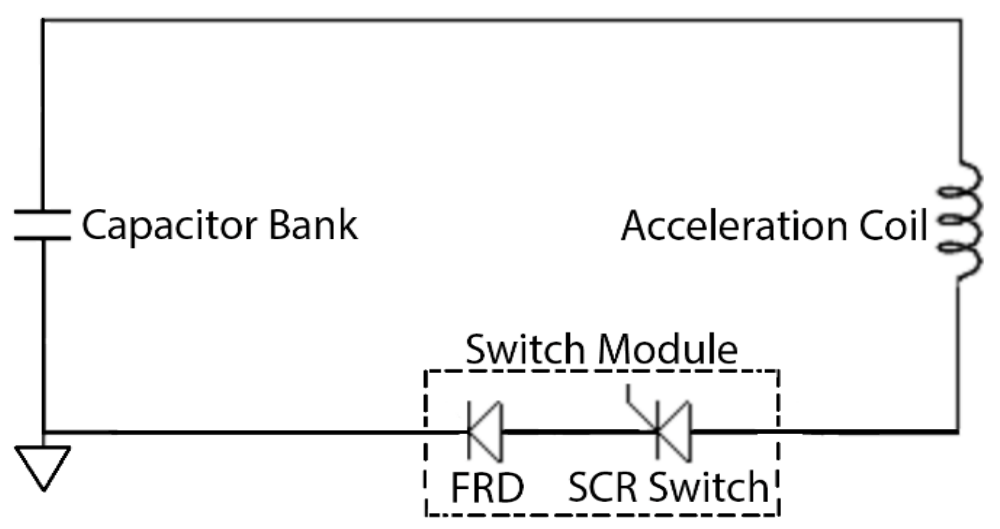

Figure 1. Simplified IPPT circuit schematic showing the circuit with a series SCR switch and FRD. suggests that the devicecan withstand currents in a $10 \mu$ s pulse of up to $400 \mathrm{~A}$. The diode is placed in series with an SCR switch, which is a Dynex ${ }^{\mathrm{TM}} \mathrm{PT} 85 \mathrm{QW}$ x45, optimized for pulsed power applications. The switch is rated for a maximum reverse blocking voltage, $V_{R R M}$, of $4.5 \mathrm{kV}$ and a mean forward current, $I_{F, A V}$ of $1670 \mathrm{~A}$. Connecting each diode into the circuit in the same manner yields the same circuit losses for each diode, including conduction and

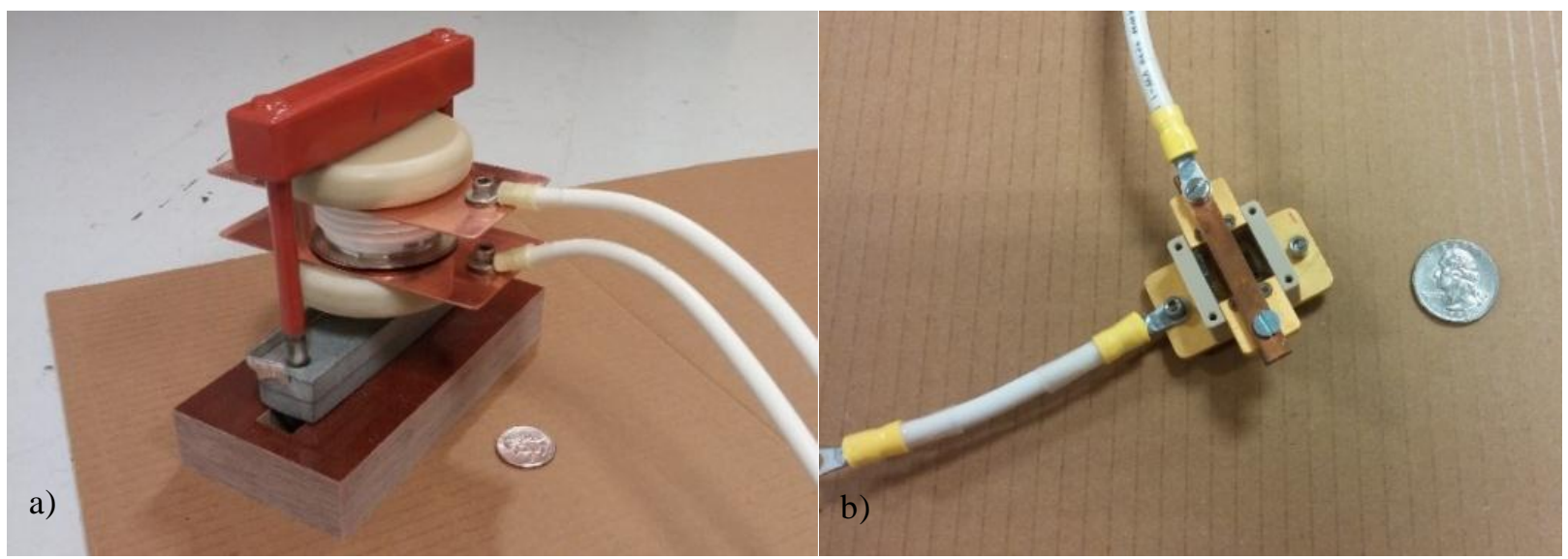

Figure 2. a) The $5.5 \mathrm{kVSi}$ fast diode from ABB is mounted in a Wakefieldclamp with a compressive force of approximately 16 kN. b) A 5.8 kV SiC PiN diode from Cree, Inc.

switching losses of the main s witch and FRD. This makes the recapture efficiency our best metric for an equivalent, quantitative comparison between the two diodes.

In other applications, it has been shown ${ }^{9}$ that $\mathrm{SiC}$ devices have a superior reverse recovery res ponse as compared with similarly rated Si devices. The wider bandgap of the material results in a breakdown voltage 10 times higher than that of $\mathrm{Si}^{10}{ }^{10}$ making it an ideal material for high voltage applications. In addition, the non -linear relationship between the drift region thickness and breakdown voltage allows for much thinner drift regions in wide bandgap devices, resulting in lower conduction voltage drop ${ }^{11,12}$ and less time needed for reverse recovery due to a lower volume of excess charge carriers. $\mathrm{SiC}$ also has a high thermal conductivity as compared to other semiconductor materials, and can therefore operate at higher temperatures. Further information on the $\mathrm{SiC}$ material properties and its subsequent incorporation into solid-state device designs can be found in Ref. 11. These characteristics make SiC devices highly promising for IPPT applications as we might expect a SiC diode to result in higher circuit recapture efficiency than what can be obtained using a conventional Si diode. However, SiC device manufacturing is a relatively new process ${ }^{13}$, and validation testing must be performed to determine how they will perform in highpower pulsed circuit applications. 


\section{Testbed Thruster Design and Construction}

A modular, small-scale IPPT was designed and fabricated at NASA Marshall Space Flight Center (MSFC) to serve as a flexible testbed for solid state s witching components and pulsed propellant injection technologies. As these components are integrated and tested in the device, it will eventually permit tes ting in repetition-rate mode, as opposed to the single-shot testing that has historically been completed in other IPPT development projects. ${ }^{14}$ Thruster design specifications and main subsystem fabrication for the bench-top testing configuration are discus sed in the following section. Additional information on the testbed thruster design and fabrication can be found in Ref. 14.

The testbed thruster was fabricated using a combination of custom and commercial off-the-shelf (COTS) components. Of the five major subsystems, only three are assembled and integrated for the preliminary bench-top testing described in this paper. These are the acceleration coil, the capacitor bank, and the switch module. A preionizer and pulsed propellant injection valve are not necess ary for the electrical switching tests and we re not installed for the present work. The thruster electrical parameters are summarized in Table 1.

Table 1. Testbed thruster electrical circuit parameters.

\begin{tabular}{|c|c|}
\hline Eectrical Parameter & Nominal Value \\
\hline Energy, J/pulse & 80 \\
\hline Pulse Length, $\mu \mathrm{s}$ & $\sim 20$ \\
\hline Charge Voltage, $\mathrm{kV}$ & Up to 4 \\
\hline Peak Coil Current, $\mathrm{kA}$ & $>12$ \\
\hline Capacitor Bank, $\mu \mathrm{F}$ & 650 \\
\hline Coil Inductance, $\mathrm{nH}$ & 200 \\
\hline Circuit Stray Inductance, $\mathrm{nH}$ & 10 \\
\hline Predicted Circuit Stray Resistance, $\mathrm{m} \Omega$ & Selectable: 10,20, r 30 \\
\hline Switch Type & Silicon-controlled rectifier \\
\hline
\end{tabular}

The primary materials used in the prototype testbed fabrication are Lexan, phenolic, and fiberglass rod. A Lexan frame provides the structure on which all thruster components are mounted. The frame is approximately $40 \mathrm{~cm}$ (15.75 in) wide, $40 \mathrm{~cm}$ (15.75 in) tall, and $38 \mathrm{~cm}$ (15 in) long. Four $35 \mathrm{~cm}$ (13.8 in) long, $1.6 \mathrm{~cm}(0.63$ in) diameter fiberg las s threaded rods connect the two separate pieces of $1.3-\mathrm{cm}(0.5 \mathrm{in})$ thick Lexan that constitute the frame. Components are then mounted onto the rods to hold everything together.

\section{A. Acceleration Coil}

The acceleration coil, shown in Fig. 3, consists of six 10 gauge copper coil wire leads connected in parallel to the remainder of the pulse circuit. The flat coil geometry was chosen because it is relatively simple to fabricate and has been used for previous IPPTs, including the PIT and the Faraday Accelerator with Radio-frequency Assisted Discharge (FARAD) thrusters. ${ }^{1,2,14}$ The copper coil leads are placed in grooves machined in a Lexan coil form, where each path is in the formof an Archimedes spiral. The inner diameter of the coil is $5 \mathrm{~cm}(2 \mathrm{in})$, and the outer diameter is $15 \mathrm{~cm}(6 \mathrm{in})$. Each lead starts on the front side of the Lexan coil form, spirals radially outward toward the edge of the pattern, passes through a hole in the Lexan to the back side of the coil, and then spirals radially inward to a point on the backside that is directly opposite from its starting point.

During actual thruster operation, the mutual inductance between the acceleration coil and the plasma current sheet varies as the plas ma is accelerated away from the coil. However, in the present work data are presented only for the electrical circuit parameters corresponding to the case where the "plasma" is infinitely far from the coil face. Practically speaking, the plasma is at a

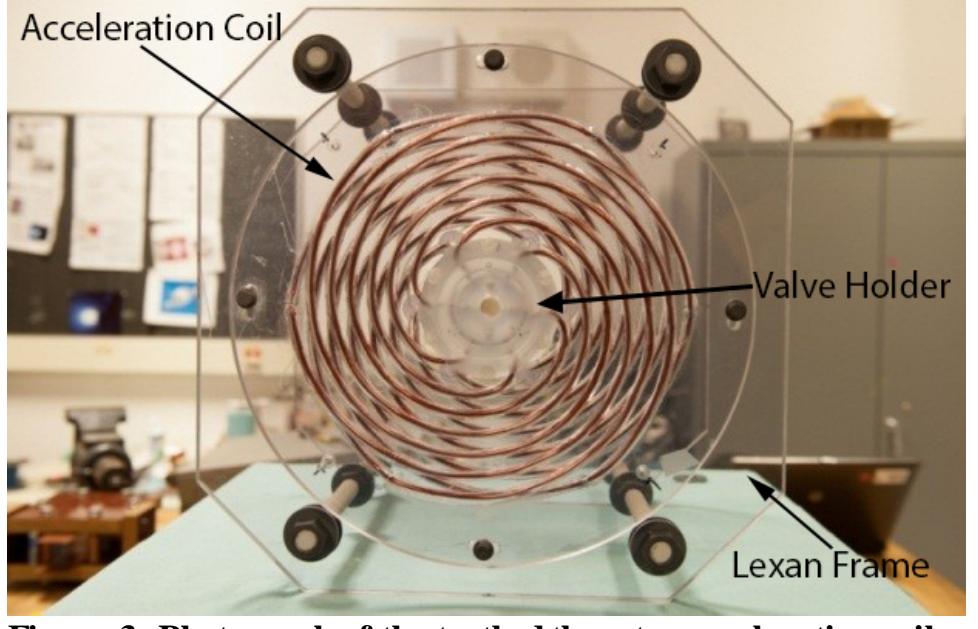

Figure 3. Photograph of the testbed thruster acceleration coil. 
distance much greater than the coil's electromagnetic decoupling distance, $z_{o}$, such that the mutual inductance between the coil and plasma currents has reduced to zero. The coil inductance, $L_{c}$, was measured as approximately $700 \mathrm{nH}$. For completeness, the decoupling distance for the coil, $z_{o}$ is approximately $6 \mathrm{~cm}$.

\section{B. Capacitor Bank}

Energy is stored in a capacitor bank (Fig. 4) and then discharged rapidly through the acceleration coil. The capacitor bank consists of three oil-filled, vacuumcompatible capacitors manufactured by CSI Capacitors. The total capacitance of the bank can be approximately 10,20 , or $30 \mu \mathrm{F}$, depending on how many of the capacitors are connected in parallel. For the bench-top testing described here, only one $10 \mu \mathrm{F}$ was used. The measured capacitance was approximately $9.88 \mu \mathrm{F}$.

\section{Switch Module}

The switch module was assembled in several configurations to permit a comparis on of the turn-off, or circuit clamping, capability of two different power diodes to each other, and also to a baseline, no-diode configuration. The ceramic compression ("hockey puck") package SCR is clamped with an applied force of $40 \mathrm{kN}$ $( \pm 10 \%)$. The mounting yoke that is used to clamp the SCR consists of two 2.54-cm (1-in) thick phenolic slabs held together by six $13.97-\mathrm{cm}$ (5.5 in) long stainless steel bolts. Phenolic tubes are used to electrically insulate the bolts (Fig .5). To facilitate tightening of the bolts, sockets are welded to a metal plate to hold the module in place as the bolts were tightened with a torque wrench to the necessary compressive load. The thruster circuit stray inductance and series diode serve to limit the current and voltage rise rate through the SCR during switching, eliminating the need for a protective snubbercircuit. The diodes are connected to the copper tabs clamped to either side of the SCR.
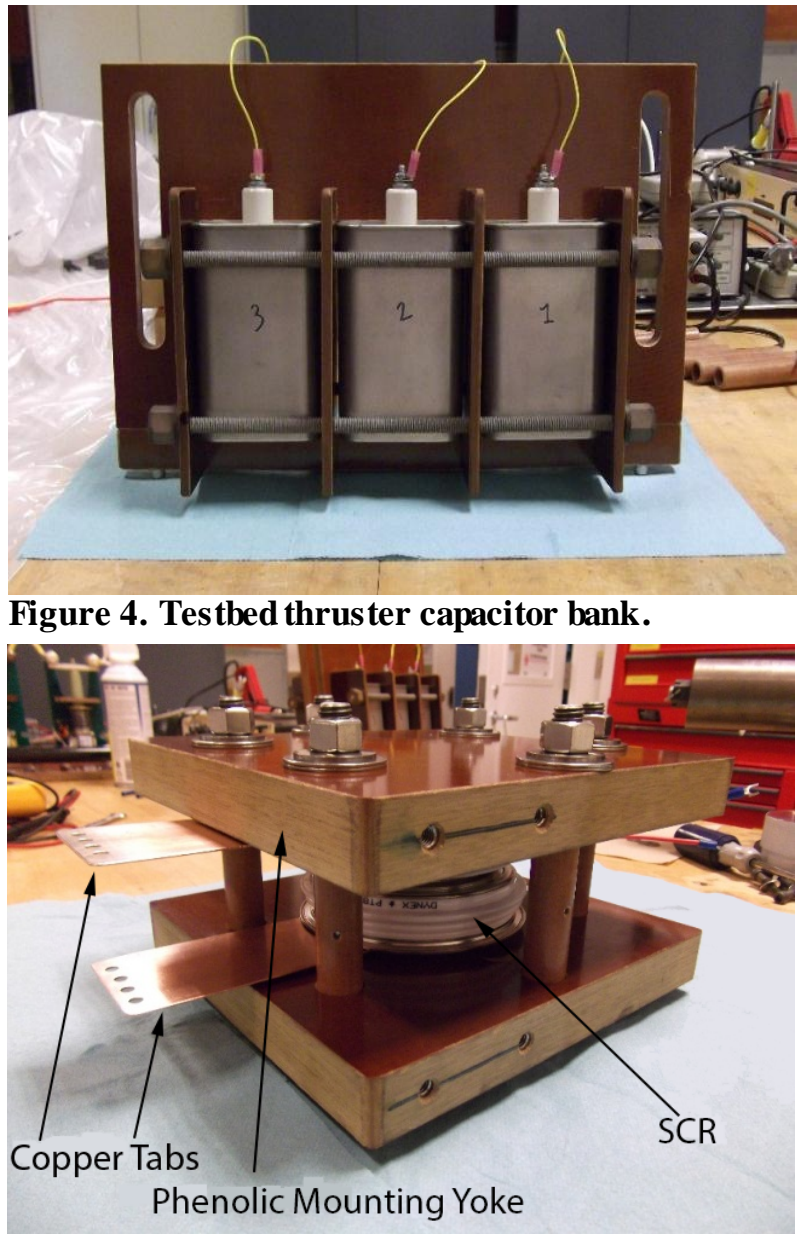

Figure 5. Ass embled SCR s witch module.

\section{Experiment}

The as sembled testbed thruster is shown in Fig. 6. Switching waveform measurements were recorded with no series diode in the circuit to obtain baseline unclamped waveforms. The measurement was repeated using one $\mathrm{Si}$ diode and two separate SiC diodes, in turn. Two SiC diodes of the same type were tested to determine whether performance was consistent between the two prototype devices. The circuit schematics and measurement locations for the testing are shown in Figs. 7a and 7b. The loop current is measured using a Pearson current monitor and the voltages across the switch and diode are measured using Tektronix differential high voltage probes. The SCR is activated by a $20 \mu$ s trigger pulse applied to the gate using a custom driver circuit.

The current and voltage waveforms collected for a s witch module configuration containing only the Dynex SCR with no series FRD provide a baseline against which to evaluate the performance of the $\mathrm{Si}$ and $\mathrm{SiC}$ diodes in the IPPT. To minimize the risk of damaging the SCR with no diode protection from a high reverse voltage rise rate in place, the circuit in this configuration was operated at a relatively low charge voltage. The collected loop current and switch voltage waveforms for a nominal charge voltage of $250 \mathrm{~V}$ are shown in Figure 8a, while the capacitor voltage, $V_{C}(t)$, is shown in Figure $8 \mathrm{~b}$. The measured loop current $i(t)$ is used to calculate $V_{C}(t)$ as

$$
\mathrm{V}_{\mathrm{C}}(\mathrm{t})=-\frac{1}{C} \int_{0}^{\mathrm{t}} \mathrm{i}(\mathrm{t}) \mathrm{dt}+V_{C i}
$$


where $C$ is the capacitance and $V_{C i}$ is the initial capacitor charge voltage. We observe that the SCR conducts even after the initial current zero crossing, and does not permanently return to a blocking state after the $20 \mu \mathrm{s}$ trigger pulse is removed. This implieds self-triggering of the SCR, likely caused by high values of reverse votage rise rate in the absence of the series FRD. A curvefit of the loop current waveform indicates a total circuit inductance and resis tance of approximately $1.24 \mu \mathrm{H}$ and $58.6 \mathrm{~m} \Omega$, respectively. The frequency of the waveform is found to be slightly greater than $45 \mathrm{kHz}$. For an unclamped configuration with no series FRD, all or nealy all voltage is assumed to be removed from the capacitor bank following a single ringing discharge cycle. In this case, $\eta_{r}$ will be very small or zero.

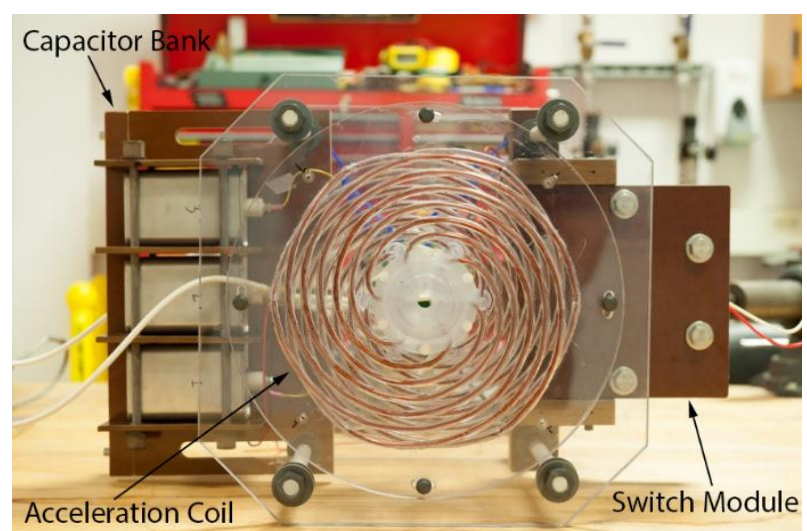

Figure 6. Assembled testbed thruster showing the acceleration coil, capacitor bank, and s witch module.

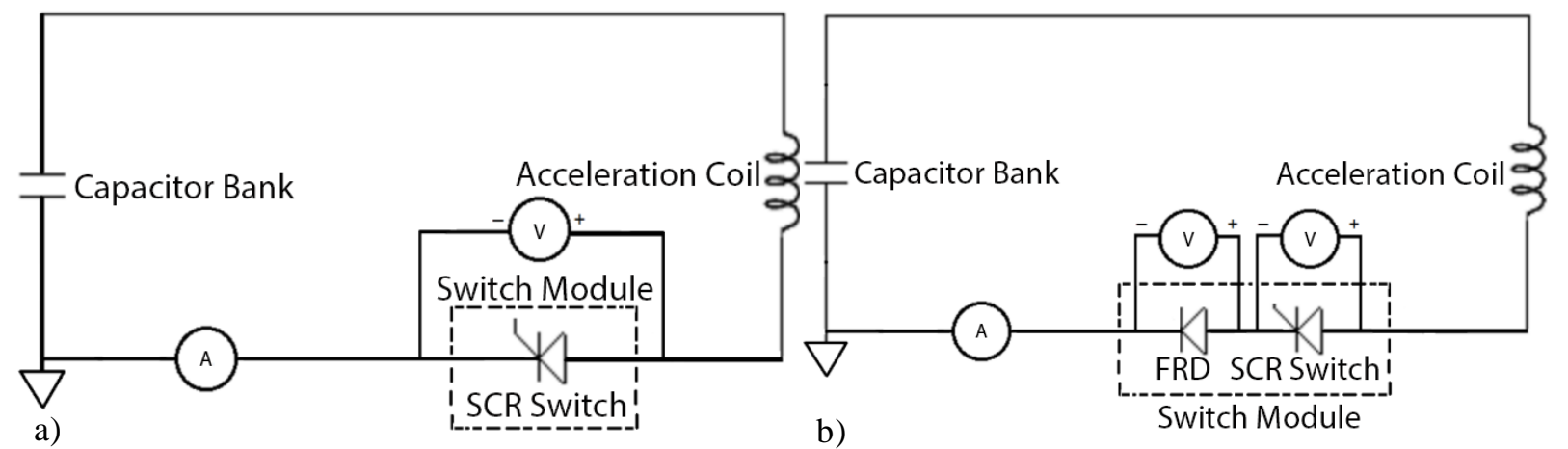

b)

Figure 7. IPPT circuit configurations tested: a) Bas eline no-diode configuration. b) Circuit configuration used to test the Si and SiC FRDs.
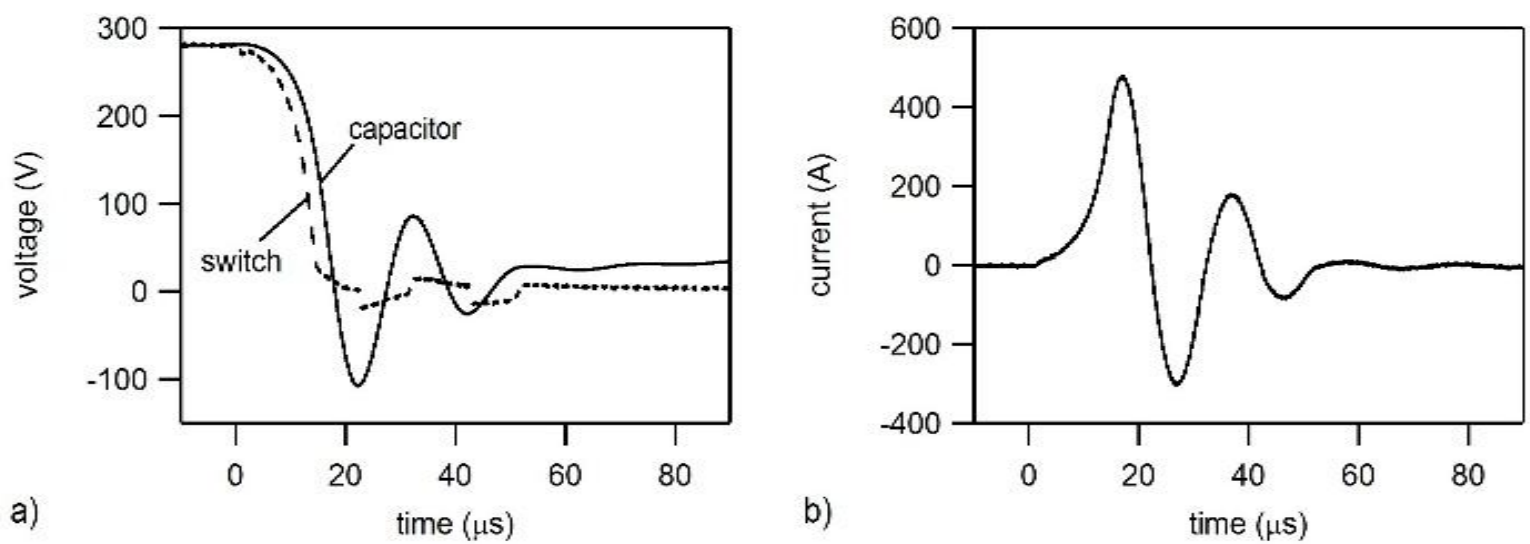

Figure 8. Bas eline configuration waveforms at a nominal charge voltage of $250 \mathrm{~V}$ : a) meas ureds witch voltage and calculatedcapacitor voltage and (b) loop current.

Switch voltage and loopcurrent waveforms are collected for the Sidiode and two SiC diodes of the same type. Meas urements of both SiC diodes yielded very similar results, so only the waveforms for one are presented. Figure 9 shows a comparis on between the calculated capacitor voltage and loop current for the Si and SiC diode configurations at a nominal charge voltage of $550 \mathrm{~V}$.

The reverse recovery response is observed in the loop current waveform following the first zero corssing. A zoomed-in view of the reverse recovery waveforms (Fig. 9c) clearly shows that the SiC diode experiences a shorter 
reverse recovery time and lower magnitude reverse recovery current than the Sidiode. This relationship is found to hold for each charge voltage tested, as well as for the second SiC diode.

\section{A. Recapture Efficiency}

The capacitor voltage waveforms for the $\mathrm{Si}$ and $\mathrm{SiC}$ diodes, corresponding to nominal charge voltages of $300 \mathrm{~V}, 350 \mathrm{~V}, 450 \mathrm{~V}$, and $550 \mathrm{~V}$, are used to calculate the percent recapture efficiency as

$$
\eta_{r}=\frac{E_{C f}}{E_{C i}} \times 100 \%
$$

where $E_{C i}$ and $E_{C f}$ represent the initial and final capacitor bank energies, respectively. These are calculated as

$$
E_{C i}=1 / 2 C V_{c i}^{2}
$$

and

$$
E_{C f}=1 / 2 C V_{c f}^{2}
$$

where $C$ denotes the capacitance and $V_{C i}$ and $V_{C f}$ are initial and final capacitor bank voltages. The recapture efficiencies for each circuit configuration are summarized in Table 2.

\section{B. Diode Reverse Recovery}

From the measured waveforms, it is apparent that there is a significant difference between the reverse recovery response of the $\mathrm{Si}$ and $\mathrm{SiC}$ diodes. The diode turn-off time is critical to the performance of the clamped IPPT circuit topology. For each diode, the magnitude of the reverse recovery response increases with increasing forward current. This is due to the greater amount of charge in the intrinsic region at turn-off. ${ }^{12}$ The turn-off response can be quantified by computing the reverse recovery parameters, including peak reverse current, $I_{d R M}$, reverse recovery time, $t_{r r}$, and total reverse recovery charge, $Q_{r r}$. These are estimated from the reverse recovery waveform using a triangular appoxiamtion of the reverse current area (Fig. 10). Time $t_{o}$ represents the time of the initial current zero crossing, while $t_{r r}$ is the total time necessary for the diode to transition froma conduction state to a blocking state. The magnitude of $I_{d R M}$ indicates the maximum reverse current seen by the device during turn-off. The triangular approximation calculates the total $Q_{r r}$ as the area of the shaded region, where $I_{d R M}$ estimates the height of the triangle and $t_{r r}$ estimates the length of the base.

The total diode $Q_{r r}$ is an important measure of the device turnoff time, in that the greater the charge stored in the in trinsic region at turn-off, the more time is necessary for the diode to transition from a conduction state to a forward blocking state. The charge must either be swept out of the region by an electric field, or recombine with other charged particles in the material. Longer turnoff time often implies high switching power los ses and stress on circuit components. The parameters for all three diodes are tabulated in Table 3.
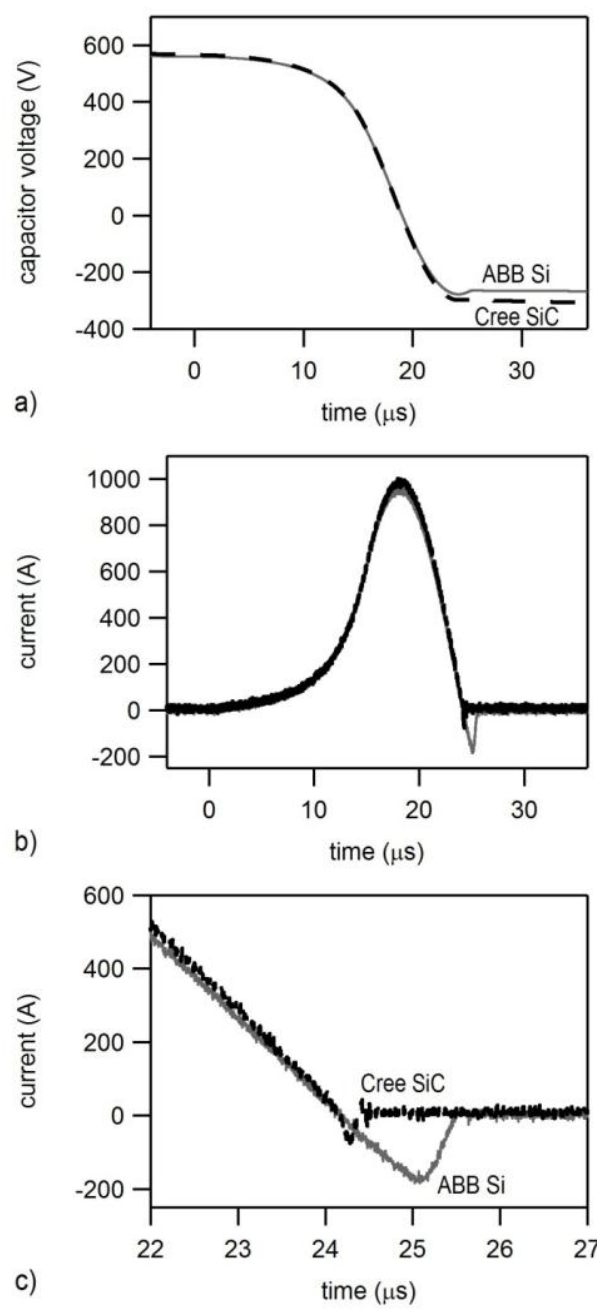

Figure 9. Comparison data collected for $\mathrm{Si}$ and SiC FRDs: a) calculated capacitor voltages, b) complete time-history of the loop currents, c) A zoomed-in view of current at reversal.

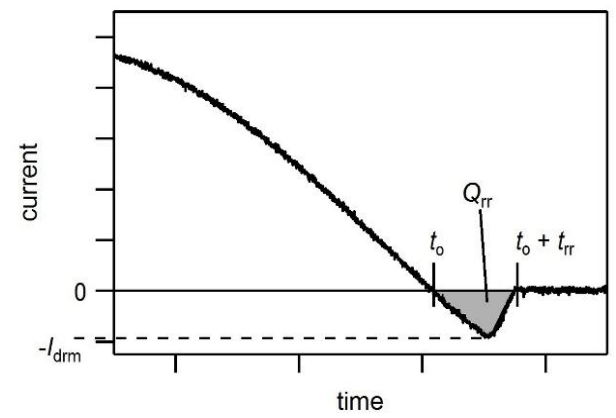

Figure 10. Graph showing how reverse recoevery parameters are defined. 
Table 2. Tabulated test conditions and recapture efficiencies for the ABB Si diode and the two Cree SiC diodes.

\begin{tabular}{|c|c|c|c|c|}
\hline Device Under Test & Nominal Charge Voltage $(V)$ & $\begin{array}{l}\text { Initial Capacitor } \\
\text { Energy, J }\end{array}$ & $\begin{array}{l}\text { Final Capacitor } \\
\text { Energy, J }\end{array}$ & $\begin{array}{l}\text { Recapture } \\
\text { Efficiency, } \\
\%\end{array}$ \\
\hline \multirow[t]{4}{*}{$A B B S i$} & 300 & 0.399 & 0.040 & $10.025 \%$ \\
\hline & 350 & 0.630 & 0.070 & $11.111 \%$ \\
\hline & 450 & 1.10 & 0.200 & $18.182 \%$ \\
\hline & 550 & 1.55 & 0.350 & $22.581 \%$ \\
\hline \multirow[t]{4}{*}{ Cree SiC 1} & 300 & 0.380 & 0.080 & $21.053 \%$ \\
\hline & 350 & 0.590 & 0.123 & $20.847 \%$ \\
\hline & 450 & 1.08 & 0.270 & $25.000 \%$ \\
\hline & 550 & 1.59 & 0.460 & $28.931 \%$ \\
\hline \multirow[t]{4}{*}{ Cree $\mathrm{SiC} 2$} & 300 & 0.3902 & 0.060 & $15.312 \%$ \\
\hline & 350 & 0.6402 & 0.092 & $14.420 \%$ \\
\hline & 450 & 1.1093 & 0.237 & $21.405 \%$ \\
\hline & 550 & 1.5260 & 0.423 & $27.739 \%$ \\
\hline
\end{tabular}

Table 3. Tabluated reverse recovery parameters for the ABB Si diode and the two Cree SiC diodes.

\begin{tabular}{|c|c|c|c|c|}
\hline Device Under Test & $\begin{array}{l}\text { Nominal Charge Voltage } \\
(V)\end{array}$ & $\mathrm{I}_{\mathrm{dRM}}, \mathrm{A}$ & $t_{\mathrm{rr}}, \mu s$ & $\mathbf{Q}_{\mathrm{rr},}, \mu \mathrm{C}$ \\
\hline \multirow[t]{4}{*}{$\overline{A B B S i}$} & 300 & 88.22 & 1.66 & 73.22 \\
\hline & 350 & 105.5 & 1.55 & 81.76 \\
\hline & 450 & 139.0 & 1.41 & 98.00 \\
\hline & 550 & 172.0 & 1.32 & 113.52 \\
\hline \multirow[t]{4}{*}{ Cree SiC 1} & 300 & 29.7 & 0.20 & 2.97 \\
\hline & 350 & 42.5 & 0.25 & 5.31 \\
\hline & 450 & 56.0 & 0.21 & 5.88 \\
\hline & 550 & 64.0 & 0.21 & 6.56 \\
\hline \multirow[t]{4}{*}{ Cree SiC 2} & 300 & 34.2 & 0.27 & 4.62 \\
\hline & 350 & 39.8 & 0.24 & 4.77 \\
\hline & 450 & 52.4 & 0.24 & 6.29 \\
\hline & 550 & 65.8 & 0.24 & 7.89 \\
\hline
\end{tabular}

The effect of the FRD on the IPPT circuit performance is evident in Fig. 11, wh ich shows a comparison between the capacitor voltage and loop current waveforms for the no-diode, Si diode, and SiC diode configurations. Clamping of the circuit energy is observed in Fig. 11a, where both diodes serve to preclude ringing. Figure 11b highlights the difference between the current reversal for each configuration.

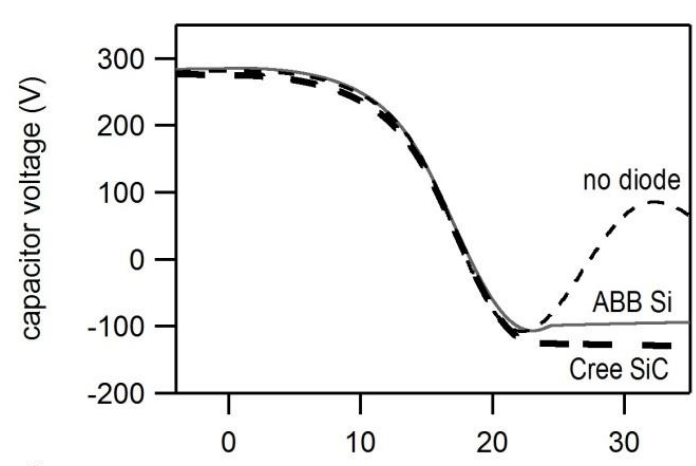

a)

$$
\text { time }(\mu \mathrm{s})
$$

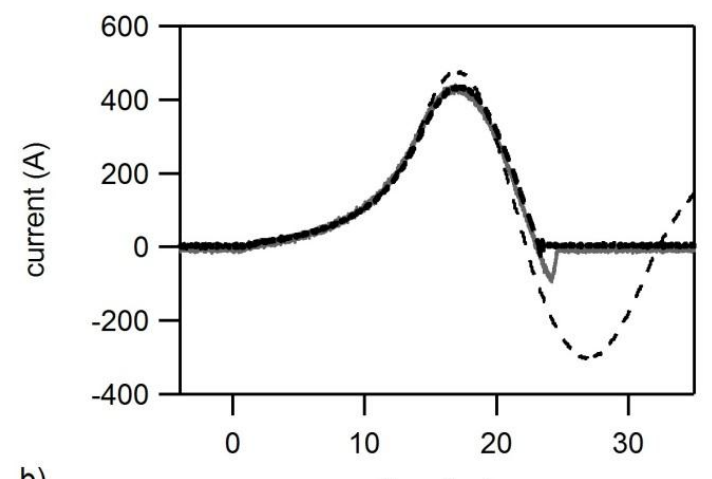

b)

$$
\text { time }(\mu \mathrm{s})
$$

Figure 11. Comparison of waveforms at $250 \mathrm{~V}$ charge voltage for the no-diode, Si diode, and SiC diode configurations.

8

American Institute of Aeronautics and Astronautics 


\section{Conclusion}

The data demonstrate that diode-clamping in an IPPT circuit permits energy recovery by halting the discharge after the first half-cycle, limiting resis tive dis sipation and leaving the capacitor bank partially charged. This has the potential to make the circuit more energy efficient and can reduce heating through lower resis tive dissipation. Both of these are significant advantages in a space environment, where the production of electricity can be costly from both a financial and mass allocation standpoint and where thermal management of power electronics is a serious concern. In addition, the comparis on of the $\mathrm{Si}$ and SiC FRDs points to a significant advantage in using fastswitching SiCdevices for this application. This point is quantified by the circuit recapture efficiency. The use of a series SiFRD in the drive circuit results in a maximum recapture efficiency of over $20 \%$ for the conditions tested, while the use of a SiC FRD results in a maximum recapture efficiency approaching $30 \%$. The data show that this difference is largely due to the superior reverse recovery response of the SiCdevices, which stems from the unique material properties of the wide bandgap material used.

\section{Acknowledgments}

The authors would like to acknowledge the management support of Mr. Jim Martin, Ms. Mary Beth Koelbl, Mr. Chad Summers, and Ms. JeriLaw. In addition, we appreciate the contributions and support of our colleagues Dr. Richard Eskridge, Darren Boyd, Doug Galloway, Tommy Reid, and Adam Kimberlin.

\section{References}

${ }^{1}$ Dailey, C. L., and Lovberg, R.H., “The PIT MkV Pulsed Inductive Thruster,” NASA CR-191155, 1993.

${ }^{2}$ Polzin, K. A. "Faraday Accelerator with Radio-Frequency Assisted Discharge (FARAD)," Ph.D. Dissertation, Mechanical and Aerospace Engineering Dept., Princeton Univ., Princeton, NJ, 2006.

${ }^{3}$ Polzin, K. A., and Choueiri, Edgar Y., "Performance Optimization Criteria for Pulsed Inductive Plasma Acceleration," IEEE Transactions on Plasma Science, vol.34, no.3, pp.945,953, June 2006

${ }^{4}$ J. Bernardes, and S. Merryman, "Parameter analysis of a single stage induction mass driver," 5th IEEE International Pulsed Power Conference, IEEE Paper PI-27, pp. 552-555, 1985.

${ }^{5}$ Dailey, C. L., and Lovberg, R.H., "Pulsed Inductive Thruster (PIT) Clamped Discharge Evaluation,” TRW Applied Technology Div., Redondo Beach, CA, Rep. AFOSR-TR-89-0130, 1988.

${ }^{6}$ General Atomics Energy Products, “The Effect of Reversal on Capacitor Life,” Engineering Bulletin 96-004, 2003.

${ }^{7}$ Polzin, K. A., "Comprehensive Review of Planar Pulsed Inductive Plasma Thruster Research and Technology," Journal of Propulsion and Power, Vol. 27, No. 3, pp. 513-531, 2011.

${ }^{8}$ Gustaveo, B., "Modeling and Simulation of Power PiN Diodes within SPICE," Ph.D. Dissertation, Electrical Engineering Dept., Polytechnic Univ. of Turin, Turin, Italy, 2006.

${ }^{9}$ Elasser, A., Agamy, M., Nasadoski, J., Bolotnikov, A., Stum, Z., Raju, R., Stevanovic, L., Mari, J., Menzel, M., Bastien, B., and Losee, P., "Static and Dynamic Characterization of $6.5 \mathrm{kV}, 100 \mathrm{~A}$ SiC Bipolar PiN Diode Modules," Energy Conversion Congress and Exposition (ECCE), 2012 IEEE, vol., no., pp.3595-3602, 15-20 Sept. 2012.

${ }^{10} \mathrm{Yu}$, L., "Simulation, Modeling, and Characterization of SiC Devices,” Ph.D. Dissertation, Electrical and Computer Engineering Dept, Rutgers, The State Univ. of New Jersey, New Brunswick, NJ, 2010.

${ }^{11}$ Matsukawa, T., Chikaraishi, H., Sato, Y., and Shimada, R., "Basic Study on Conductive Characteristics of SiC Power Device for its Application to AC/DC Converter," Applied Superconductivity, IEEE Transactions on, vol.14, no.2, pp.690,692, June 2004

${ }^{12}$ Ostling, M., Ghandi, R., and Zetterling, C. -M, "SiC Power Devices — Present Status, Applications and Fut ure Perspective," Power Semiconductor Devices and ICs (ISPSD), 2011 IEEE 23rd International Symposium on , vol., no., pp.10,15, 23-26 May 2011 
${ }^{13}$ Matsunami, H., "Progress in Wide Bandgap Semiconductor SiC for Power Devices," Power Semiconductor Devices and ICs, 2000. Proceedings. The 12th International Symposium on , vol., no., pp.3,9, 2000

${ }^{14}$ Polzin, K. A., Martin, A. K., Eskridge, R. H., Kimberlin, A. C., Addona, B. M., Devineni, A. P., Dugal-Whitehead, N. R., and Hallock, A. K., "Summary of 2012 Inductive Pulsed Plasma Thruster Development and T esting Program," NASA/TP -2013217488, 2013. 